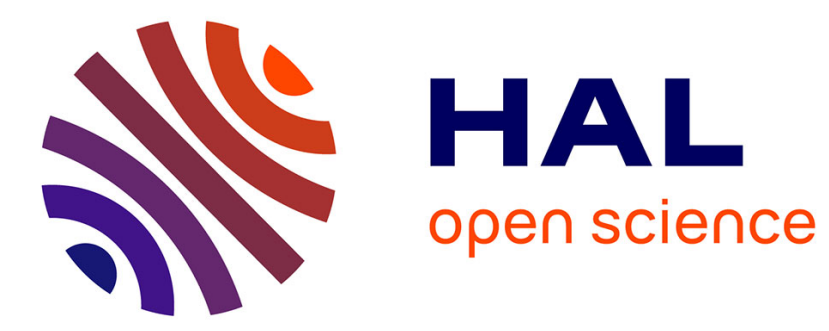

\title{
The Climate Visualizer: Sense-Making Through Scientific Visualization
}

\author{
Douglas N. Gordin, Joseph L. Polman, Roy D. Pea
}

\section{To cite this version:}

Douglas N. Gordin, Joseph L. Polman, Roy D. Pea. The Climate Visualizer: Sense-Making Through Scientific Visualization. Journal of Science Education and Technology, 1995, 3(4), pp.203-226. hal00190576

\section{HAL Id: hal-00190576 https://telearn.archives-ouvertes.fr/hal-00190576}

Submitted on 23 Nov 2007

HAL is a multi-disciplinary open access archive for the deposit and dissemination of scientific research documents, whether they are published or not. The documents may come from teaching and research institutions in France or abroad, or from public or private research centers.
L'archive ouverte pluridisciplinaire HAL, est destinée au dépôt et à la diffusion de documents scientifiques de niveau recherche, publiés ou non, émanant des établissements d'enseignement et de recherche français ou étrangers, des laboratoires publics ou privés. 
AERA SIG Education, Science and Technology

\title{
The Climate Visualizer: Sense-Making Through Scientific Visualization
}

\author{
Douglas N. Gordin, ${ }^{1,2}$ Joseph L. Polman, ${ }^{1}$ and Roy D. Pea ${ }^{1}$
}

\begin{abstract}
This paper describes the design of a learning environment, called the Climate Visualizer, intended to facilitate scientific sense-making in high school classrooms by providing students the ability to craft, inspect, and annotate scientific visualizations. The theoretical background for our design presents a view of learning as acquiring and critiquing cultural practices and stresses the need for students to appropriate the social and material aspects of practice when learning an area. This is followed by a description of the design of the Climate Visualizer, including detailed accounts of its provision of spatial and temporal context and the quantitative and visual representations it employs. A broader context is then explored by describing its integration into the high school science classroom. This discussion explores how visualizations can promote the creation of scientific theories, especially in conjunction with the Collaboratory Notebook, an embedded environment for creating and critiquing scientific theories and visualizations. Finally, we discuss the design trade-offs we have made in light of our theoretical orientation, and our hopes for further progress.
\end{abstract}

KEY WORDS: Visualization; cducational technology; project-enhanced science learning; climate; networking; telecommunications.

\section{INTRODUCTION}

This paper describes the design of a learning environment, called the Climate Visualizer, intended to facilitate scientific sense-making (Newman et al., 1994) in high school classrooms. The Climate Visualizer provides students the possibilities of crafting, inspecting, and annotating scientific visualizations (SciVs). Our focus on SciV derives from an attempt to promote students' appropriation of the practices of scientists, thus allowing for more situated learning in the manner of cognitive apprenticeship (Brown et al., 1989). SciV is both increasingly important to the practice of science and has significant potential within science education (Gordin and

\footnotetext{
${ }^{1}$ School of Education and Social Policy, Annenberg Hall, 2115 North Campus Drive, Northwestern University, Evanston, Illinois.

${ }^{2}$ Correspondence should be directed to Douglas N. Gordin, School of Education and Social Policy, Annenberg Hall, 2115 North Campus Drive, Northwestern University, Evanston, Illinois.
}

Pea, 1994). When students create and analyze SciVs, they are using the same tools and data sets as practicing scientists, thus helping them to join scientific communities of practice. This participation can also help students realize that science is a dynamic field where a primary activity is the design and analysis of theories, rather than the memorization of facts and processes (Linn et al., 1994). Through examining SciVs, students can come to perceive formerly inaccessible physical processes, thus allowing them to observe patterns and construct theories to explain those regularities. However, we believe there are serious obstacles before students can appropriate SciV. The goal of the Climate Visualizer is to provide a scaffolding for students to surmount these difficulties. These scaffolds take the following forms: Ease the task of crafting visualizations by providing powerful affordances (Gibson, 1977) for their specification and sufficient context for their interpretation; promote their use within meaning-making conversations and their use within the creation of scientific theories; foster a community of scientific 
inquiry through a shared inscriptional system that incorporates SciVs; and employ a project-enhanced science learning pedagogy (Ruopp et al., 1993) that emphasizes student-designed and -conducted research projects with guidance and facilitation by teachers and others.

The organization of the paper is as follows: First, the Introduction defines the goals of the Climate Visualizer, a description of SciV, and an example of a student project that used the Climate Visualizer. Second, in Theoretical Basis and Design Principles for the Climate Visualizer, the theoretical motivations behind our program of having students appropriate SciVs are described and the methods of scaffolding this appropriation through the Climate Visualizer are presented. Third, in Crafting Scientific Visualizations with the Climate Visualizer, the specific design of the Climate Visualizer's scaffolding support for students is scrutinized. Attention is paid to the screen layout and the provision of spatial and temporal context. In addition, the quantitative and visual representations used by the Climate Visualizer are described. Fourth, in The Climate Visualizer in the Classroom Environment, the larger context of use within classrooms is described. This includes a discussion of how project-enhanced science learning can promote student motivation and interest and student collaboration through a shared inscriptional system.

\section{What is Scientific Visualization?}

SciVs are colorful pictures, formed from abstract data, that often attempt to appear similar to the phenomena being represented. An example of such a visualization is a color map of the world where the color indicates the temperature. A rainbow spectrum is employed, where blue stands for cold and red for warm, much as it is on television weather reports. We produce SciVs such as these with the Climate Visualizer by using archival data from the National Meteorological Center (NMC) through a widely distributed CD-ROM. Several features of this example are salient. First, color is used evocatively to indicate varying magnitudes, where our prior associations with these colors correctly anticipate their use. Second, an abstract quality, namely temperature, is illustrated visually. Third, the spatial distribution of the data is indicated through the use of space in the visualization, thus not requiring separate references to connect temperature values to spatial locations, as would be required by tabular data. Fourth, the data have been processed through models achieving an even distribution or gridding of data values. Fifth, the data are rendered by high speed computer graphics.

The techniques of SciV have become increasingly popular and have proven useful to scientists in the pursuit of inquiries into realms with spatial and temporal components and also in providing a compelling inscriptional system to present and discuss models and theories. Applications of SciV in atmospheric science, medicine, materials science, fluid dynamics, and astronomy have become commonplace (Borroughs, 1991; Brodie et al., 1992; McCormick et al., 1987; Kaufman and Smarr, 1993).

\section{Student Inquiry Aided by the Climate Visualizer}

The example below shows some of the ways we have already seen students finding and pursuing a scientific question with the Climate Visualizer. This story has been reconstructed from field notes of participant observers at a workshop in the summer of 1993. The use of the Climate Visualizer by high school teachers and students during this weeklong workshop at Northwestern University provided us with formative evaluation for the software and pedagogical approach described in this paper. The Climate Visualizer has not had intensive use in high schools as of this writing; however it will be used in several classes at two Illinois high schools starting in the autumn of 1993. In this example, some minor details have been changed for purposes of clarity, and the actual names have been changed. The story involves two high school students (Keith and Bob) working with the Climate Visualizer, a high school teacher (Stan), and a workshop facilitator (Gregg).

Gesturing to a colorful map of the Northern Hemisphere, where temperature was depicted as colors and the altitude of pressure ${ }^{3}$ as contour lines, Keith asked, "Why is the altitude lower at the Poles than near the equator?" This thoughtful question followed an earlier debate over whether a high altitude implied high or low pressure at the surface. That debate, between several high school students and educators, had concluded altitude and pressure were correlated, so a high altitude indicated a high pressure zone. During their conversation, the white

\footnotetext{
${ }^{3}$ The "altitude of pressure" refers to the height of a constant pressure level above sea level. Pressure is commonly used as an independent variable and altitude as a dependent variable in me-
} 
board in the classroom-sized workroom had become full of illustrative diagrams as various metaphors were tried.

Keith's question evoked a new eruption of conversation as new conjectures were expounded that explained the altitudes he had seen in the Climate Visualizer screen. "The atmosphere is thinner at the North Pole, so the altitude of pressure has to come down lower," explained Gregg, one of the workshop facilitators. "No, it's that the air at the North Pole is colder so it stays close to the surface. That's what causes the low pressure," countered Stan, a high school teacher.

The debate continued until lunch with different hypotheses being advanced and illustrated. Bob, another high school student, exclaimed, "Arguing about these ideas has been great, it's fun to try and figure out what altitude means here." This discussion took place early in the week of our formative evaluation of the Climate Visualizer. Like many questions brought up, it might have not come up again, but Keith had been struck by this question and wanted to see it through. Later in the week, when the students were given time to develop a project, Keith knew exactly what he wanted to investigate. He enlisted his fellow student Bob in the project, and the two of them started by writing down their question and their conjecture.

Question: Why is the altitude of pressure higher at the Equator and lower at the North Pole?

Conjecture: Near the North Pole the air is colder, so it is closer to the surface of the earth. At the equator the air is warmer, so it rises higher above the surface of the earth.

Getting the question and conjecture written down took a while. First, they had to remember exactly what the question and conjecture were and then they had to find the words to express them. After they had written it, Bob said, "We're done now, so can we do something else?" Gregg encouraged them to go on by saying, "You've expressed your question and your conjecture, but what support do you have for it? Are you sure that the North Pole always has a lower altitude? If it does, how can you check if your conjecture has support?"

\footnotetext{
teorology and climatology. To understand the altitude of pressure, it might be instructive to think of reversing the relationship, namely the pressure of altitude, for example, the pressure at 10,000 feet. Over an area this pressure varies, depending on where large masses of air and clouds move. The altitude of pressure picks a given pressure, say $500 \mathrm{mb}$, and measures the altitude between it and sea level. The extent of this height too
}

Spurred by this challenge, Keith and Bob used the Climate Visualizer to query SciVs at the North Pole and near the Equator to find specific altitudes and temperatures and entered them into a spreadsheet that Gregg helped them design. The table they constructed had columns for the temperature and altitude at the North Pole and near the Equator at two different pressure levels, $850 \mathrm{mb}$ and $200 \mathrm{mb}$; they filled it in by simply clicking at the locations on the SciVs and then reading the values off the screen. They then calculated the differences in temperature and altitude between the two locations at the different pressure levels and the percentage change that these differences represented. After they had laboriously put the data together and added it to their notebook, Bob asked, "Are we done now?" Pushing them still further, Gregg prompted, "But does your data support or refute your conjecture?" Keith responded indignantly, "It supports it, of course. Well, at least for the winter months." "So, now add a section explaining the connection between your data and your conjecture." Keith then changed the conjecture to refer only to winter and described how their conjecture explained the lower altitudes of pressure found at the North Pole.

At the end of the workshop, Bob and Keith presented their work to the whole group of students, teachers, and workshop facilitators. They began their presentation by explaining their question and showing the visualizations that had prompted it. They then presented their conjecture, the data they had collected, and explained why their data supported their conjecture. Afterwards one of the teachers stopped Bob and congratulated him on a good job. Bob thanked him and then exclaimed, "But what we did was only the beginning, this project could go on for weeks. We have to figure out what is happening during the rest of the year too. There's a lot more data to collect and interpret, before we've really finished this project."

This interaction has several important features. Despite their lack of knowledge about atmospheric science, Keith and Bob were able to construct SciVs similar to those used by practicing atmospheric sci-

varies depending on air masses. Note that in both these cases altitude and pressure are positively correlated, that is, when either independent variable increases so does the corresponding dependent variable. The primary reason that "altitude of pressure" is commonly used is that high altitude winds tend to travel along constant pressure surfaces, thus they travel along contour lines showing the altitude of pressure. 
entists. The Climate Visualizer also helped them interpret the SciVs by providing an explanatory context. The SciVs provided an opportunity for sense-making conversations about the altitude of pressure; further, in subsequent conversations they used the SciVs to express their conclusions. Hence, scientific inquiry involving the design and defense of theories was facilitated by the Climate Visualizer, allowing students to specify and complete a project of their own choosing. We now turn to our attempt to provide a theoretical background and motivation for these goals.

\section{THEORETICAL BASIS AND DESIGN PRINCIPLES FOR THE CLIMATE VISUALIZER}

To present the theoretical underpinnings of the Climate Visualizer as a learning environment that aids in science education, the question "What is science education?" must be answered. The first step to answering this question is to state, in broad strokes, our view of education and, second, to discuss how this view is particularized when the subject of education is science.

Our basic view of education is that it consists in the acquisition and critique of the values, language, and practices of a culture. In short, education is acquiring and critiquing cultural practices. This view of education is founded in the work of several philosophers, psychologists, and educators. One of the most foundational thinkers in this account is the philosopher Wittgenstein (1967), who describes speaking and writing as participating in "language games" that include not only words, but their physical and social contexts. Hence, learning language is to join a cultural system that has complex rules governing how, when, and why words are used. Similarly, Pea (1993b) has emphasized the distributed nature of our thought, coining the term "distributed intelligence." This account also emphasizes the interactions of material artifacts and collaboration in the accomplishment of activities. A particular focus of this work is on the role of human desire and design in building environments that support our activities.

Writing directly about the processes of education, John Dewey (1956) emphasized "learning by doing," where students would perform jobs similar to those performed by the adults in their surrounding society. In the terms introduced above, the students could be said to be acquiring, through practice, the "language games" of these fields or appropriating the "distributed intelligence" present in their society to perform tasks. Recent accounts have used apprenticeship as a model for contemporary education (Lave, 1990; Lave and Wenger, 1991; Collins et al., 1989). These thinkers are not simply calling for a resurgence of apprenticeships in place of our contemporary schooling; rather, they seek to use the exemplar of apprenticeship in reforming our current schooling system. One characteristic of apprenticeship to retain is connecting student activities with the practices of an adult community - Lave has called this becoming peripheral members of a community of practice. However, the students need not emulate all the practices or beliefs of the adult community, since the priorities and needs of students differ from adults; specifically, the goal of the adults is often to produce some artifacts (e.g., producing many cars, or solving a scientific problem), whereas the goal of the students is to learn. In emphasizing this distinction, we can adopt an example from Dewey where he notes that when a student is learning to weave it is not as important that the student produce many spools of cotton, rather the student should come to understand the experience of a weaver and the activity of weaving, including the strengths and limits of its enabling technology, its scientific basis, and its social organization.

Given a theoretical orientation towards learning that emphasizes learning as participation in a community of practice, it is natural that in answering the question, "What is science education?," we turn to examine the scientific community, since it is this community that contains the values, language, and practices we would like students to appropriate, insofar as they further students' learning. For this account of the scientific community, we turn to the descriptions and analysis of sociologists of scientific practice. These sociologists have provided an account of what scientists do when they "do science" that emphasizes the roles that external inscriptions play and a characterization of why various inscriptions are successful in scientific discourse (Latour and Woolgar, 1979; Latour, 1988; Lynch and Woolgar, 1988). Two methodological assumptions underlie much of this work and serve to illuminate why it differs from many conventional accounts of scientific practice. First, sociologists of science engage in a practice of anthropological strangeness. This refers to a conscious suspension of preconceptions and knowledge about the role of scientists in society, the 
meaning and purpose of the inscriptions that scientists use, and the epistemological status of the products of science. Second, as mentioned above, sociologists assume that the cognitive and perceptual mechanisms employed by people are roughly the same independent of both culture and time. Hence, the abilities of scientists do not rely on uncommon insight or a qualitative jump in intellect.

In this sociological characterization of science the various formulas, diagrams, pictures, and textual accounts have been collectively labeled inscriptions. These inscriptions play a pivotal role in the actions of scientists. We will only briefly review these claims and their implications here. Vast amounts of work go into the production of them; the preparation for one inscription uses others; and debate over issues raised in these inscriptions dominates routine conversations in scientific workplaces. "Science" is thereby defined in these studies as the creation of these inscriptions and the social practices that accompany their creation and use. In particular, two characteristics of inscriptions describe their use and success in the scientific enterprise. First, these inscriptions are used in argumentation as persuasive devices that can be used to compel agreement among peers. For example, a conversation could center on the quickest way to traverse a maze. In order to demonstrate a solution, a map of the maze is drawn with different paths shown in different colors and the lengths of the paths are annotated. This inscription then becomes a powerful tool in resolving the debate.

Second, various formal and material aspects of inscriptions imbue them with power. These attributes of inscriptions are enumerated as: immutable, mobile, flat, scalable, reproducible, recombinable, superimposable, the ability to be placed in written texts, and mergeable with geometry (Latour, 1988). While we will not review accounts and definitions for these attributes here, these qualities can again be seen in the map of the maze discussed above when it is drawn on paper. Indeed, the enormous utility of paper for achieving these goals becomes an important factor in the success of inscriptions. Thus, paper becomes a common medium that permits various inscriptions to be combined, referred to, and accumulated. This reconstruction of science by sociologists of science continues onto the epistemological status of inscriptions. Whereas scientists frequently ascribe a basis in nature for their inscriptions, this sociological account emphasizes their construction in practice through a layering of inscriptions or representations. Using the maze example above, the specialized map that is constructed builds on accepted inscriptions of maps for describing spatial relationships, and also builds on representations in mathematics for summing distances. This example underscores the observation that representations are overlaid and built upon after they have become widely accepted. In addition, an effective inscription often relies upon resemblances with the object it represents. These resemblances are crafted, and as such, their use may undergo substantial development, as was the case with realistic painting. The view of scientific material thus changes from a reflection of nature, as some theories of science would have it, to the products of craft or even a manufacturing process.

The sociological account of science serves to emphasize the role of inscriptions or external representations in entering into the scientific community of practice. It posits that such inscriptions play a necessary role in the formation of any major scientific enterprise. Hence, the appropriation of these inscriptions provides important opportunities for students to increase their access to scientific practice. It is important to emphasize that appropriating these inscriptions does not involve merely manipulating their syntactic forms. Insofar as these inscriptions are embedded in "language games" or "distributed intelligence," their contexts of use must also be appropriated. The potential for separating inscriptions from their customary usage has been examined by Schoenfeld (1987) in the case of mathematical problem solving, where he found that while students may employ the same formal systems as mathematicians, they deployed them in a very different manner. In particular, the students were found to concentrate on syntactic and extrinsic characteristics, rather than on the substantive meaning. Hence, while our consideration of science education has led us to a decision to appropriate scientist's inscriptions, there is the need to examine how to appropriate, not only the external representations or the "words," but also to appropriate their context of use.

The practice of "learning conversations" (Pea, 1992 ) is instrumental in understanding how students can come to appropriate inscriptions, that is, to come to understand their appropriate context of use within science and not merely manipulate their surface syntax. The defining characteristic of a learning 
conversation is the negotiation of meaning, rather than the one-way transmission of knowledge. Within learning conversations, the meanings of words or inscriptions and the occasions for their appropriate use are negotiated and socially constructed. This account of learning is largely consistent with Vygotsky's (1978) description where a learner's participation in a zone of proximal development provides the foundation for his/her subsequent developmental progress. Within learning conversations, inscriptions are used to garner consensus by providing visual referents for discoveries, conclusions, as well as disagreements. This use of inscription emphasizes its ability to act in "symbolic mediation," to use Roschelle's (1990) term, thus providing a means for knowledge and uncertainty to be negotiated. It is through the collaboration and negotiation involved in learning conversations that the "reading" of inscriptions is achieved. Within the context of a learning conversation, inscriptions are used to signify specific agreements, controversies, and relationships. Indeed, many signs are so accepted and standardized that it is difficult to imagine that they are not transparent. For example, in many cultures, a hand wave is used to signify hello and good-bye, and placing the forefinger and thumb in a circle with the other three fingers splayed out is used to signify "okay." Yet these nonverbal communications, like the verbal and written representations we have been discussing, are not transparent until they have been learned (von Glasersfeld, 1987). Many inscriptions still can benefit from meaning negotiation even after they have been extensively studied (e.g., financial models, mathematical models, tax forms, or systems dynamics models).

The above description of inscriptions has attempted to establish their centrality within science and thereby their importance to science education. However, some inscriptions are better for some purposes than others (e.g., it is easier to multiply using Arabic numbers than using Roman numbers). Borrowing on the sociological critique of inscriptions within science, we suggest the following dimensions may be used to characterize the utility of an inscription for affording a task: (1) Its capacity to support learning conversations about that task or, as stated earlier, its utility as a rhetorical or persuasive device in discussing that task; (2) the inscription's capacity to be materially and symbolically integrated, that is, does it have those qualities identified by the sociologists of science that allow it to be flexibly inter- mingled with other inscriptions?; (3) the extent to which the inscription employs familiar systems of meaning to provide assistance to its understanding (e.g., metaphors or analogies that suggest how it can be used). This reliance on prior frameworks is described in the above sociological account where inscriptions are shown to be formed by layering and combining existing inscriptions. These prior layers provide a means to understand and predict the mechanisms of the new layer, thus promoting its usage and acceptance. These criteria will be used to loosely characterize the utility of an inscription.

SciV provides an excellent example of an inscriptional system that has become central to the practice of science and exhibits the above characteristics of a useful inscriptional system. First, the increasing use and importance of SciV has been well documented in recent accounts, and SciV has been steadily increasing its share of national funding in the United States in the High Performance Computing and Communications program, as well as abroad (Borroughs, 1991; Brodie et al., 1992, D'Amico et al., 1994; McCormick et al., 1987; Kaufman and Smarr, 1993; Office of Science and Technology Policy, 1991, 1993; Short et al., 1976). Many areas of science are using SciV: biologists to map the human genome, cosmologists to show the largescale structure of the universe and galaxies, and chemists and material scientists to interrogate the landscapes of atoms and molecules. Second, they provide compelling visual images of abstract phenomena, thus providing images that promote consensus and debate. Examples include using visualizations of the ozone hole to summarize and dramatize the debate over the role of flourocarbons in altering our atmosphere, using medical sonograms of fetuses to emphasize their human-like attributes in disputes over the legality of abortion, LANDSAT satellite images of floods and forest fires to illustrate the extent of disasters, predictions showing the Earth's warming to argue for limiting carbon dioxide, and the animations of thunderstorms and other weather phenomena to predict and describe weather. Clearly, as rhetorical aids, SciVs have become enormously compelling. Third, many, if not all, of the integrative qualities enumerated by the sociologists of science are possessed by SciVs. Without going into detailed consideration of these qualities, suffice to say that a classic example of these qualities is a map and SciVs can be considered a type of map. One point of some importance to the treatment here 
is that these qualities focus on an inscription's ability to be merged, compared, and contrasted with other inscriptions. Usually, the medium for these integrations is on paper. SciVs are created and processed by computers, although they can also be transferred to paper. Hence, SciVs are inscriptions that can occupy two different mediums, within the computer and on paper. It is within the computer that they best maintain their flexible, integrative nature with other inscriptions - this integration is powerful since it allows interactive exploratory data analysis and problem solving. Thus, it is within the computer that we will mainly consider them. Fourth, SciVs build on prior representations and use familiar metaphors to facilitate their comprehensibility. The data illustrated in a SciV incorporate existing representations: the data are numeric, are in terms of accepted units, and are of some selected quality (e.g., the SciVs of the ozone hole show the number of Dobson units of ozone present). In addition, SciVs gain much of their rhetorical power from seeking verisimilitude with the phenomena being represented. For example, medical imaging renders its results in the colors that are conventionally associated with elements of the body, so blood is shown as red, skin as a flesh color, and so on. These renderings are not necessary; rather, they are chosen for the explanatory or rhetorical force that comes from using familiar metaphors and verisimilitude with phenomena. In summary, we argue that SciVs should be used by students in learning science, not only because scientists use them but also because of why scientists use them, namely, that they provide a powerful inscription that promotes learning conversations, can be flexibly manipulated, and build on existing metaphors and representations. Yet, there is a powerful reason for students to use SciV beyond those listed here, namely, doing science with SciV can help reorganize students' basic conceptions of what it means to do science.

The utility of tools to reorganize human activity and in particular for computer-based cognitive tools to reorganize education is described by Pea (1985, 1987; Pea and Kurland, 1987). This account begins with the understanding that tools and cultural institutions mediate and transform our relationships with nature, our society, and ourselves. Cognitive tools or technologies do this by augmenting and reorganizing our mental abilities. Through these reorganizations the essential practices involved in performing a task can change. We argue that SciV can have this type of impact on the practice of learning science. The current practices in learning science often center around the memorization of facts and processes, thus leading to the belief that science is a static discipline whose problems are solved. When students practice science by engaging in SciV activities, they can look for patterns in data, form theories around these patterns, and attempt to confirm these theories using the data that formed the SciVs. The proportion of human practices in a given content domain, once cognitive tools are developed and appropriated in use, often shift toward higher-order strategic and exploratory activities rather than clerical, symbol-manipulation-like tasks. SciV is no exception to this pattern. This opportunity to engage in exploration of phenomena that was previously inaccessible shows the utility of technology for promoting exploration and the design of theories to explain what is found. The anecdote that begins this paper shows several students collaboratively engaging in a similar process. These activities can lead to a view of science as dynamic and on-going, where knowledge is constructed, debated, and tested (Linn et al., 1994). It is this reorganization of student practices and beliefs that provides the best argument for including SciV within the practices of high school science. This is not to imply that these changes can occur solely by using SciV: Only part of the process of restructuring science education can be accomplished through cognitive tools, the rest rely on social supports.

In appropriating SciV for the high school classroom we seek to enable students to learn its "language game" or for it to become part of the "distributed intelligence" (Pea, 1993a) with which they accomplish tasks. It is useful to discuss which changes can be accomplished by cognitive technologies and which through social supports. In analyzing the functions of cognitive technologies for teaching mathematics, Pea (1985) distinguishes between purpose/motivation functions and process functions. The functions we hope to perform with cognitive tools are process functions, not motivation. In particular, these process functions include conceptual fluency or the automatic performance of routine tasks, exploration or discovery learning, and linking representational systems. Providing motivation and other purpose functions (i.e., metacognition and problem-solving skills), we hope to engage using social supports. The primary focus of this paper is to show how the cognitive technology called the Cli- 
mate Visualizer aids students to use SciVs through scaffolding these process functions. This is done by automating the creation of SciVs so that students can easily create and modify SciVs, making explicit tacit knowledge that scientists use to read SciVs, and providing operations on SciVs that can be used to further investigate data and to test theories. In the section Crafting Scientific Visualizations with the Climate Visualizer, specific design decisions are motivated in terms of these goals. Other aspects of using SciVs are left to the social supports. In particular, social supports are used to motivate students to pursue inquiry, to help students understand which projects or questions SciVs can be used to answer, to help them accomplish and assess their projects, and to help them use SciVs as effective rhetorical devices in expressing questions or theories. The social supports designed to meet these objectives include collaborative projects and access to scientists. A discussion of these social supports is provided in the section The Climate Visualizer within the Classroom Environment.

\section{CRAFTING SCIENTIFIC VISUALIZATIONS WITH THE CLIMATE VISUALIZER}

The theoretical perspective described above presents us with the challenge of enculturating high school students into meaningful use of SciV. In order to achieve this goal, we needed to first identify how atmospheric scientists use SciV and then find ways of creating affordances that could allow students to create and understand SciVs, similar to those that scientists use. As part of a broader study of the atmospheric science community of practice (D'Amico et al., 1994), we were able to examine in depth one climatologist's use of SciV with the NMC data set. Professor Raymond T. Pierrehumbert introduced us to the NMC data set by showing how SciVs formed from it illustrate basic climatic processes (e.g., how hot air masses flow from the equator towards the poles and cold air masses move from the poles toward the equator) and by allowing us to observe and participate in an undergraduate course on climate where students manipulated this and other data sets to aid in solving problem sets. This formative study included making video and audio tapes of Pierrehumbert's manipulations of SciVs. Our analysis of these sessions and records allowed us to identify substantial tacit knowledge that was embedded in the creation and interpretation of the SciVs, as well as standard "moves" in the "language game" of SciV. These moves included visually exploring SciVs by changing color palettes and compressing or expanding the range of values that are mapped into the palette (this will be explained below in more detail in the section Visual Representations) in order to find patterns, form conjectures, and provide evidence for theories. We have designed the Climate Visualizer to help high school students (like Bob and Keith) similarly explore and craft SciVs. This goal has dictated that powerful affordances be provided and that they be arranged in a manner that explicitly suggests when and how they should be used.

Figure 1 presents one of the Climate Visualizer views ${ }^{4}$ that we have designed to make explicit scientists' knowledge and suggests their accepted use. In essence, this screen presents a dynamic SciV, in that it provides for the specification and inspection of SciVs. We discuss the coordination and design of these affordances below in terms of the layout of the Climate Visualizer's screen; the important representations it uses, both quantitative and visual; and the potential difficulties for students. In addition, the relationship of the design to the goals laid out above is made explicit throughout.

\section{Description of the Climate Visualizer Screen}

This section discusses the overall appearance of the Climate Visualizer. In particular, we cover the following topics: the layout of the Climate Visualizer screen; the ways in which the SciVs are placed into spatial, temporal and geographic context; and a description of how multiple SciVs can be compared.

\section{Layout of the Climate Visualizer Screen}

The layout of the screen has been carefully designed to promote students' ability to easily construct SciVs. The screen layout attempts to lead the student's focus of attention by employing a standard English-style text-informed arrangement that is organized from top to bottom and from left to right. The use of this spatial arrangement has been shown to increase a diagram's intelligibility (Winn and Holliday, 1982). Overall, the screen is vertically divided

\footnotetext{
${ }^{4}$ Views provide for specific representations of variables on a specific data set. They are discussed in more detail below.
} 


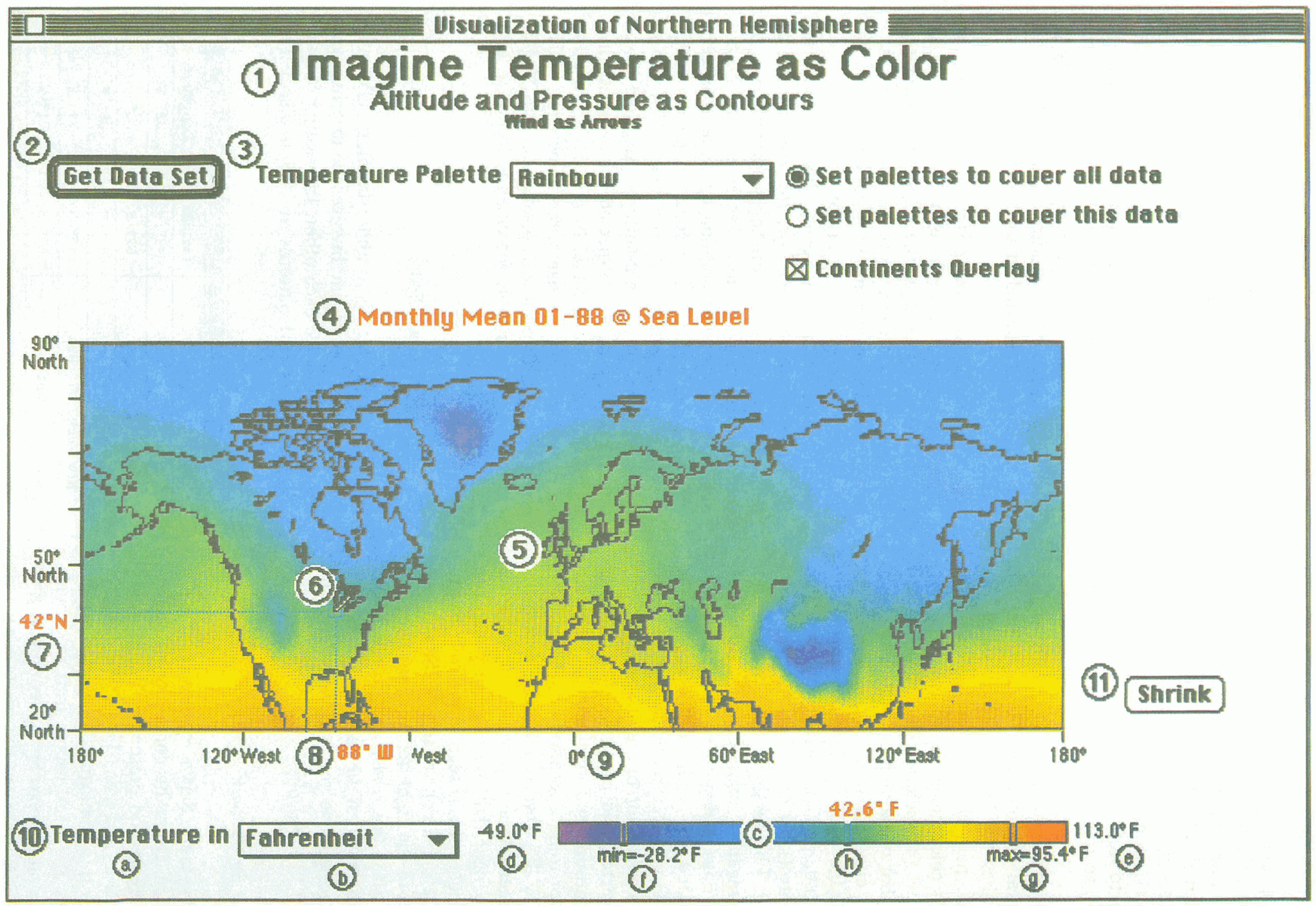

Fig. 1. The Climate Visualizer's main window: (1) title of view - describes the basic representations this view may display; (2) Get Data Set-provides the controls for obtaining the SciV for a specific date; (3) means to manipulate the SciV's use of color palettes and overlays; (4) title of SciV - describes the date, time, and variables currently displayed; (5) current scientific visualization; (6) query box - indicates the currently selected grid point; (7) latitude of current query; (8) longitude of current query; (9) background grid showing latitude, longide, and locil time at selected longitudes; (10) display palette; (a) variable shown in this display palette; (b) units popup - shows currently selected units for this variable and allows switching to other units; (c) current palette for this variable; (d) value of minimum color for this palette; (e) value of maximum color for this palette; (f) value and position of minimum value in this data set; ( $\mathrm{g}$ ) value and position of maximum value in this data set; (h) value and position of the current query selected on the SciV (indicated at point 6 ); (11) Shrink button - allows user to shrink current SciV and display palettes into a small window. 
into the following four sections: the title (i.e., Imagine Temperature as Color ...), a row of controls, the SciV with its title centered underneath, and the bottom region for display palettes that show data values that correspond with selected areas within the SciV. This vertical arrangement provides an organization from top to bottom, providing a movement from general to specific and from controls to their result.

We now describe the items more fully to analyze the horizontal organization. The title, or topmost line, describes the basic representations this view may display; namely, temperature can be encoded as a raster color image, altitude as contours, and wind as arrows (or vectors). These are standard representations that climatologists employ; as such, by automatically choosing them, we seek to make the tacit knowledge of the scientists explicit and automated for students. The next row, on the left, provides the controls for obtaining the SciV for a specific date (Fig. 3 below shows this dialogue) and, on the right, the means to manipulate the SciV's use of color and overlays. Below these controls is their result, the requested SciV with a title describing the date, time, and variables currently displayed. In addition, a box appears on top of the image to indicate the currently selected grid point, with callout lines to indicate the latitude and longitude of the point; in Fig. 1, the approximate location of Chicago, Illinois, $42^{\circ}$ north by $88^{\circ}$ west, is selected. Hence, the horizontal arrangement also provides an organization from left to right that moves from general to specific. In particular, this is seen in the row of controls where the leftmost control selects the data set and the controls to the right act to change the appearance of the SciV; this is also seen in the display palettes, where the name of the variable and its units are shown on the left and the current palette and values are shown on the right.

\section{Providing Context for the SciV}

Like all diagrams, SciVs require context for their interpretation. Often this context is left implicit when scientists create and present SciVs (D'Amico et al., 1994; McGee and Pea, 1994). We have attempted to make this contextual knowledge explicit by using overlays and background grids to provide spatial and temporal context. Spatial context is provided for the NMC data set via an overlay that shows the continents and through a surrounding grid of longitude and latitude numbers (e.g., Keith and Bob were able to assess quickly the locations of the North Pole and the equatorial regions by reference to the overlay and grids). Temporal context is provided for twice-daily data via a grid that shows a clock with the local time every four hours or $60 \mathrm{de}-$ grees of longitude - this feature allows students to assess quickly what time of day it is on the different regions of visualizations. Otherwise, it is tempting to fallaciously assume temperature at different parts of the earth were collected at the same local time of day.

Geographic knowledge is often prerequisite to understanding the patterns of temperature, wind, and altitude featured in the Climate Visualizers' data sets. Correlatively, there are many opportunities for incidental learning of geography as the importance of geographic location and features on climate is understood. However, when too much information is put into overlays, the visualization of data can be obscured, so further geographic context could be provided via tables. Tables provide the additional benefit of easy addition by students of geographic context that is important to specific issues they are investigating - if Bob and Keith found some locations in the mid-latitudes that had lower altitude of pressure than expected, for instance, they might add them to a table. In our design, a table entry would include a name and a location, which could be selected to show its position on a SciV. For example, a generic table for cities is shown in Fig. 2.

\section{Comparisons Between SciVs}

A primary means to understanding SciVs and the data they represent involves comparing and contrasting them to each other. Interesting or puzzling contrasts and patterns are often discovered

\begin{tabular}{|l|c|c|}
\hline \hline City & Latitude & Longitude \\
\hline Chicago, IL, USA & $42^{\circ} \mathrm{N}$ & $88^{\circ} \mathrm{W}$ \\
\hline Lond on, England & $52^{\circ} \mathrm{N}$ & $0^{\circ}$ \\
\hline Moscow, Russia & $56^{\circ} \mathrm{N}$ & $38^{\circ} \mathrm{E}$ \\
\hline New York, NY, USA & $42^{\circ} \mathrm{N}$ & $74^{\circ} \mathrm{W}$ \\
\hline Santiago, Chile & $33^{\circ} \mathrm{S}$ & $71^{\circ} \mathrm{W}$ \\
\hline
\end{tabular}

Fig. 2. Example of a geographic table - cities table. 
in this way, confounding students' expectations and challenging the limits of their knowledge, thus leading them to formulate theories or models to explain what they have observed. Particularly useful examples using the NMC data sets are comparing color images of temperature from two successive days, comparing the temperatures of winter and summer monthly means, comparing two SciVs of a single data set using different color palettes (to see the differing patterns that the palettes bring out), and comparing SciVs, which show wind as vectors, from different vertical levels of the atmosphere on the same day. The facility to do comparisons is straightforwardly available through provision for multiple smaller windows that contain SciVs. When a SciV has been crafted to completion, it can be shrunk into a smaller window (smaller versions are created by the "shrink" button, see Fig. 1). This smaller window shows the same SciV, reduced to $75 \%$ of original size. The title providing context is maintained along with its display palettes, overlays, and grids. The controls to change the SciV are not provided, as the intent of this window is to store a single SciV for comparison with other SciVs. For instance, Keith and Bob could have used these small windows to compare the altitude of pressure in winter and summer months. A "reactivate" button is provided to restore a small image to the main display in order to allow changes to its appearance.

Another way that SciVs can be directly compared is by subtracting one from another. The resulting difference clearly shows how they differ. This operation is especially useful in building theories of how changes occur: the theories can be expressed in terms of producing the observed differential. Supporting this operation (and not others) was based on our empirical investigations of how climatologists explore these data sets (D'Amico et al., 1994). Singling out and simplifying this operation encourages students to use it in their explorations of the data. During our formative evaluation, students and teachers requested a more general form of comparison, namely, animations. The creation of an animation would involve picking a start and stop date along with a sampling interval. To ensure meaningful comparisons, a single color palette across a single numeric range would be used. These animations would provide another powerful means to detect patterns across time.

\section{Quantitative Data and Its Representations}

Underlying SciVs are data sets containing vast quantities of numbers. While the Climate Visualizer can be used with a wide variety of data sets, it is particularly focused on climatic and meteorological data sets. The salient characteristics of these data sets are that they cover three dimensions of space (although the Climate Visualizer will only show a two-dimensional slice) and they are characterized by multiple variables, such as temperature and wind.

Our discussion of quantitative data takes place in several sections: the method of selecting a data set to visualize; a discussion of the data types that the Climate Visualizer currently supports, described through a taxonomy of data sets; supported operations on data sets; and the methods by which specific data values can be queried.

\section{Selecting Data to Visualize}

A primary goal of the Climate Visualizer is to simplify the selection of the data sets from which the SciVs are generated. The data sets are selected through definitional attributes, such as the time when the data were collected and the qualities or variables that were measured. The view displayed in Fig. 3 shows a dialogue where an NMC data set can be selected by choosing the date and time the data were collected and by specifying a single independent variable along with one or more dependent variables. The NMC data sets include several decades of data collected twice a day covering temperature, altitude, and wind at five different pressure levels (i.e., $850 \mathrm{mb}, 700 \mathrm{mb}, 500 \mathrm{mb}, 250 \mathrm{mb}$, and $200 \mathrm{mb}^{5}$ ), as well as temperature and air pressure at sea level, and monthly means of each of these variables for the same period. Retrieving the data corresponding to these selections can entail considerable processing, since the data sets are stored in several large files on a CD-ROM. ${ }^{6}$ Like other de-

\footnotetext{
${ }_{5}^{5}$ The abbreviation for millibars is $\mathrm{mb}$, the standard meteorologic unit for air pressure. Average surface air pressure is around 1000 $\mathrm{mb}$, so $850 \mathrm{mb}$ corresponds to the point below approximately $85 \%$ of the weight of the atmosphere. Readings at $1000 \mathrm{mb}$ are rarely used in large-scale climatology because of common surface distortions to temperature and other variables due to buildings, hills, and other surface-level anomalies.

${ }^{6}$ The data currently supported are all archival data from 1964 to 1989. The NMC data sets are not made available in real time or near-time, but the archives are updated periodically. These will be added to the set available in the Climate Visualizer over time.
} 
velopments of cognitive technologies provided by computer tools (Pea, 1985, 1993b; Rheingold, 1985), the Climate Visualizer has automated these lengthy operations (i.e., provides conceptual fluency) so as to focus the students' energy on interpretive, rather than mechanical, operations.

To illustrate what we mean by such automation, it is informative to contrast what we have seen scientists do to the actions that Keith and Bob took in order to produce SciVs of temperature and altitude at $850 \mathrm{mb}$ on January 1, 1988, at Midnight GMT, using the NMC data set. A scientist would run a specialized program that extracts the data from the CD-ROM and saves the files to disk. The files are encoded by the date, time, and variables they contain - for example, the temperature data are in a file called T850010188.00, for temperature at $850 \mathrm{mb}$ on $01 / 01 / 88$ at 0 GMT or midnight. These files are then rendered by the commonly used Spyglass Transform visualization program through a series of commands to create color raster images, create contour maps, overlay one onto another, and add an overlay of the continents. Needless to say, this long sequence of actions is prone to error and requires experience and a clear understanding of the end goal. Keith and Bob, on the other hand, had only to make the selections shown in Fig. 3 from the Get Data Set dialogue in the Climate Visualizer, and the image was created, thus saving them the burden of learning many irrelevant details and encouraging their strategic examination or exploration of more SciVs.

\section{Representations of Quantitative Data}

Various taxonomies of data sets have been advanced in the effort to normalize the storage of data and to establish a consistent taxonomy (Brodie et al.,
1992). A major division in these taxonomies is between "point" data and "gridded" data. Point data provide information on specific locations that need not occur at regular intervals. In contrast, gridded data provide data at consistent intervals. Each data point can be seen to provide a value for a fixed region of space around it; hence, the entire space has values assigned to it. In this sense, the grid of data provides values for the entire space, whereas point data only provide values for a finite set of points. Other taxonomic divisions occur in the dimensionality of the space where the data are plotted (i.e., the number of coordinates necessary to specify a point) and in the dimensionality of the points themselves. These dimensions are normally categorized as one (i.e., scalar), two, three, or $n$ dimensions. Given this taxonomy, we can specify the type of data sets that the Climate Visualizer currently supports: gridded data, within a two-dimensional space or plane, where the data is scalar or two-dimensional. The motivations behind these selections were in part to use the standard scientific representations and to choose those that were practically accessible. Issues of practicality included the difficulty for students in comprehending three-dimensional images, which we do not address in this work, and the capabilities of our hardware and software platform (see Appendix for more details). Associated with the different types of data sets are different standard visual representations. We briefly present those here, but defer discussion of their use to the section below entitled Visual Representations. Scalar gridded data occurring in a plane can be shown via a raster image or a contour map. Raster images display the magnitude of a data value as a color or pixel value. Contour maps divide the magnitudes of the values into a small number of intervals and then place a bounding line around data values belonging to the same interval. Two-dimensional data occurring in a plane is

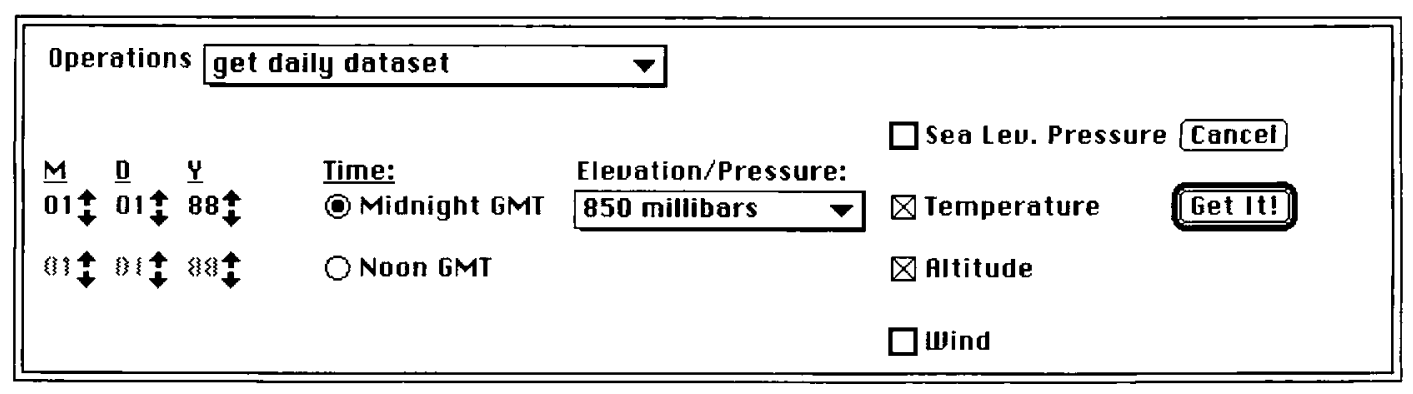

Fig. 3. Get visualization dialogue box. 
shown through vectors. The size and direction of the vectors are used to encode the pair of data values.

In the future, we plan on adding support for point data, within a two-dimensional space or plane, where the data are scalar or two-dimensional. Scalar point data occurring in a plane can be represented as a scatter plot where the magnitude of the data is represented through the size of the dot that is shown. An example of such data would be the concentration of a pollutant in the soil at selected points in an area.

\section{Operations on Data Sets}

A more diverse set of SciVs is possible if the student can create new data sets by specifying operations to be performed. For example, seasonal change can be visualized by subtracting the temperature of a day in January from a day in July. However, there is the danger that the student can specify operations that are nonsensical (Confrey, 1990); for example, an altitude data set could be subtracted from a temperature data set, thereby subtracting meters from degrees. This clearly does not produce meaningful results. Our tentative solution is to specify custom operations as part of a specific view for specific data sets. This allows the operations to be specialized for different data sets and for different representations, once again off-loading some of the cognitive work so that higher-level interpretive efforts can be made more easily by students (Pea, 1993b). The example above, of seasonal difference, is one such operation provided for the NMC data set. In addition, some general reducing operations could be provided that work on a single data set, for example, averages and standard deviation.

\section{Querying Data}

The values at a given point can be queried by clicking on that point using a tool, called the "query tool," which looks like an eye-dropper, to "pick up" the points' values. The coordinates of the point are shown as labeled callout lines to the surrounding axes. For example, when selecting a point, with the NMC data set, the latitude and longitude of the point is displayed. The values of the current dependent variables are marked with boxes on the display palettes below the SciV (see Fig. 1). Dragging the mouse across the SciV presents an animation of how these values change as the selected value on the display palette is dynamically updated. Similarly, the keyboard arrow keys can be used to move the selection point vertically or horizontally. This form of querying permits exploratory searching of the data set, for example, to find local or global minima and maxima. In addition, selecting an item from a geographic table, such as a city, positions the query tool at the appropriate location.

All of these dynamic linkages of representations are designed to provide semantic frameworks to support students in interpreting the SciV data, which are left implicit in the current scientific visualization tools used in the scientific community. In particular, the display palettes indicate the units of the data sets being displayed along with the color selected and the data value at that point. This highlights the connection between color and a value with attached units. The meaning and presence of units is something that is particularly hidden in the use of SciVs by scientists - often the units are not made explicit anywhere, even though knowledge of them is necessary for meaningful interpretation. However, while the units are explicitly labeled and the student can switch between types of units (e.g., feet and meters), this does not suffice to explain their meaning to students. This level of interpretation is left to the social surround with the SciVs acting the part of symbolic mediators in providing a specific inscription around which questions can be asked and answers negotiated.

\section{Visual Representations}

This section describes the visual representation of data within the Climate Visualizer, and details our reasoning behind our choice of representations as well as problems we anticipate. The description includes the following specific topics: our reasons for the use of standard collections or views of visual representations, the projection of the (approximately) spherical Earth onto the computer display, the visual representations used with gridded and point data sets, and the use of color.

Views Encapsulate the Practices of Scientists. Students do not have the ability with the Climate Visualizer to arbitrarily choose the visual representations that are used for a data set. Rather, they can choose between views. A view prescribes the visual representations for a data set based on the variables being represented. The views are designed 
to encapsulate the combination of visual representations that scientists use when exploring a given issue. For example, Fig. 1 presents a view of the NMC data set where temperature is represented as a raster image, and if altitude or pressure were selected, it would be shown as a contour map, while winds would be shown as vectors. An alternative view would show the magnitude of winds (by specifying only the magnitude, the wind data set is scalar rather than two-dimensional) as a raster image and temperature as a contour map. Several benefits accrue from the students' use of these standardized views. These representations are the ones that scientists have found to be most useful, that is, these inscriptions have been found to be powerful means of conveying ideas and supporting debate over issues raised by those ideas. Hence, there is good reason to expect students will also find these representations useful for the same reasons in the context of learning conversations and inquiry. In addition, through these common representations students establish a common ground on which to discuss their work with scientists. In other words, these inscriptions can serve as vehicles that allow students to legitimately participate in the community of scientists which also uses these representations.

Projecting the Earth. In order to display the (approximately) spherical Earth on a flat computer screen some projection must be used. The Climate Visualizer uses a cylindrical equidistant projection. This is a very simple projection that evenly divides the Earth into regions along latitude and longitude lines. Each region is then allocated equal area. This results in considerable distortion. For example, the top row of Fig. 1 is actually a single point: the North Pole. Hence, a student might believe a cyclone is growing as it moves North of the equator. However, this seeming growth is merely an artifact of the spatial distortion that the projection introduces. These distortions have led us to recommend that a globe be used along with the Climate Visualizer so as to provide a more accurate framework of area and shape.

In addition, we have designed two tools to help students experiment with the distortion: a distance tool and a zoom tool. The distance tool would allow the student to select two points. The distance between the points would then be shown and the shortest path between them drawn. On a spherical surface, like the Earth, the shortest dis- tance between two points lies along the great cir$\mathrm{cle}^{8}$ that connects them. Hence, the shortest path would appear as a curved line on the projection, except when both points lie on the same longitude (since all lines of longitude form great circles). These curved shortest paths should provide a clear signal to the student that the standard rules that govern Euclidean planes (e.g., the shortest distance between two points is a straight line) do not hold here. The student's investigation of shortest spherical distances could be extended through the zoom tool, which would provide a facility to magnify part of the visualization, thus obscuring the rest. The zoom tool would be used by clicking and dragging over the rectangular region to be magnified. Once zoomed, the position and size of the magnified area would be indicated by a rectangle within a thumbnail representation of the entire SciV; the SciV could be unzoomed to display it in its entirety. Sufficient zooming would change the appearance of the lines that the distance tool draws. In particular, if sufficient magnification were applied, the curved line along the great circle would appear like a straight line. Hence, by varying the level of zoom and by observing the distance lines between points, students could observe the manner in which the spherical geometry of the Earth is represented (and distorted) by the cylindrical equidistant projection. These tools provide opportunities to explore and discuss the representational conventions, that is, they provide for symbolic mediation.

The distance and zoom tools have many potential applications besides providing a mechanism to understand the distortions introduced by projections. In particular, students could use the distance tool to measure the size of phenomena being displayed, thus aiding in the creation and assessment of theories (e.g., the size of cyclones could be measured to see if their size varies proportionally with their latitude), and the zoom tool could provide the ability to focus on a particular region of interest, thus discerning greater levels of detail. Additional detail could be seen if the range of the palette were changed to the minimum and maximum data values within the magnified portion. This facility is discussed further below in the section on color.

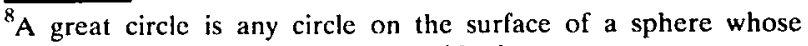
diameter intersects the sphere's midpoint.
} 
Visual Representations for Gridded Data Sets. The underlying quantitative representations of the data set determine what visual representations can be used, as was discussed above in the section on Quantitative Data and its Representations. In particular, the Climate Visualizer displays scalar gridded data sets as either raster images or contour maps. For example, Fig. 1 shows temperature from the NMC data set being visually represented as a raster image. Raster images are formed by mapping a value to a color. Thus, the number of colors determine the resolution of the map. This process is discussed in more detail below in the section on color. Similar to the assignment of colors in the raster image, contouring divides the data set into a set number of intervals. Lines are then drawn between points that lie in the different intervals. The most familiar use of contour maps is in topographic maps that use contours to denote the surface elevation; for the NMC data set, altitude lines of a pressure surface are represented as contours. Two-dimensional gridded data use vectors or arrows as their visual representation. For example, a two-dimensional data set displaying wind represents the magnitude of the wind through the size of the arrow and the direction of the wind by the direction that the arrow points. These representations are standard within the scientific community, hence providing them to the students scaffolds their acquiring the "distributed intelligence" with which scientists construct their theories.

Use of Color in Visual Representations. Color is an essential component of SciVs. Within the Climate Visualizer, color is used for the raster images and the contour maps. The colors to be used are specified via a color-palette, which is composed of an ordered list of 254 colors. Associated with a color palette are maximum and minimum data values. These values are used to create a mapping from any number to a color. Numbers below the minimum or above the maximum data value receive the first or last color, respectively. The range of numbers between the minimum and maximum values are divided evenly into 254 intervals, thus mapping the numbers between the minimum and maximum values to a color. This mapping is used to assign colors when a raster image is generated from a scalar gridded data set. The lines of a contour map are assigned colors in a similar way.

The control of color thus lies in the selection of the color palette and the minimum and maximum values that direct how the color palette is used to map numbers to colors. It is within these aspects that much of the art of SciV lies. Choosing different color palettes produces wildly different visual results. Similarly, effective choices for the minimum and maximum values of a color palette mean the difference between an image that is a single solid color versus one that displays intriguing patterns that exhibit every shade of the color palette. An important point is that a given color palette can produce very different images with different minimum and maximum values.

The Climate Visualizer provides 16 different color palettes. Examples include a rainbow color palette, a gray-scale color palette, and a color palette based on the colors assumed by heated metal ranging from dull black to fiery red. Many of the color palettes do not range evenly between two shades, rather they have sharp discontinuities between colors, a phenomenon referred to as banding. Banded palettes interweave dark shades, thus producing a contouring effect within the raster image. These color palettes were developed by the National Center for Supercomputing Applications at the University of Illinois at Urbana-Champaign and have been found widely useful, thus demonstrating the usefulness of color palettes for supporting the human task of visually identifying significant patterns in data from diverse subject matter disciplines.

Any of the available 16 color palettes can be chosen to render the raster images and contour maps. However, there are default color palettes for each variable. For example, the NMC data set uses the rainbow color palette as the default for the temperature raster image and the gray-scale color palette as the default for the altitude contour map.

The minimum and maximum values of a given color palette have default values that are specified by the view of the data set. Hence, each independent-dependent variable pair has preset minimum and maximum values. Typically, these values provide a range that covers most of the range of a variable. There are several advantages to this scheme. First, the student does not have to initially set the values, instead they can be confident that the range is set so as to ensure a reasonable SciV will be produced - this is another example of supporting access to useful representations by constraining choice space in interface design. Second, a standard range ensures a consistent framework for comparison across multiple visualizations. For example, the 
temperature range in the NMC data set is from $-45^{\circ} \mathrm{C}$ to $45^{\circ} \mathrm{C}$, so if a student examines a SciV of the temperature in January and another of the temperature in July, the colors have a consistent interpretation between the two SciVs. This allows the two SciVs to be directly compared with each other. Otherwise, when different color palettes are used or different ranges are used for the same color palette, the same number will be mapped to different colors, substantially complicating the feasibility (or sensibility) of any comparisons.

However, keeping the range of the color palette fixed comes at a cost, namely, for a single data set selection, less of the color palette will be used. Using the above example of a day in January, none of the temperatures in the NMC data set are liable to be above $20^{\circ} \mathrm{C}$. Hence, a large section of the colors is not used. These unused colors can be reclaimed through changing the minimum and maximum of the color palette to be the same as the minimum and maximum data values found in the data set. Assuming a standard distribution of values, all colors would then be shown. Yet, as noted above, if the minimum and maximum values were set in this way for a day in January and then set again for a day in July, the mappings of their values would be different; hence any direct comparison would be misleading.

Additional opportunity to revise the range of color palettes would occur through the use of the zoom tool. After magnifying a portion of a SciV, the minimum and maximum values within that portion would be substantially less than the data set's overall minimum and maximum. The color palette could be set to use this reduced range, thereby achieving greater contrast, so as to enable the user to more readily distinguish patterns of differences. Hence, a powerful use of the Zoom Tool would be to heighten contrast by changing the color palette to reflect the minimum and maximum values of smaller areas.

Our design of the use of color in visual representations, like other aspects of the use of representations, is motivated by a desire to allow students with limited expertise to create the same kind of powerful SciVs that scientists use and to use these SciVs profitably in learning conversations and scientific inquiry. As with other design decisions, it involves striking a balance between the wide range of freedom in constructing visualizations that scientists have (which holds greater potential for nonsensical conclusions and floundering for the uninitiated), and scaffolding the construction of SciVs that are most likely to be useful. The trade-offs involved in attempting to strike this balance are further explored in the section Compromises with Authenticity.

\section{Some Difficulties in Making SciV Accessible for Learning}

Students face numerous difficulties in successfully appropriating SciVs, even after taking into account the powerful affordances and contextual reminders that the Climate Visualizer provides in its design scaffolding. This section presents some of our early findings of difficulties students have in using the Climate Visualizer's representations. These findings come out of our formative evaluation during some hours of student usage in the spring of 1993 and the week-long workshop previously mentioned, which took place during the summer of 1993. By presenting these findings, we intend to detail some of the challenges we anticipate in further design of the Climate Visualizer and/or the social surround in which the Climate Visualizer is placed.

There are numerous difficulties students might have in dealing with the quantitative representations discussed above. One of the common problems we have seen is in understanding the meaning of the variables used in the Climate Visualizer and the meaning of the units in which these variables are measured. For example, the altitude of pressure is a very difficult concept even for scientifically sophisticated adults, unless they are familiar with its use in atmospheric science, and the unit millibars, used to measure pressure, is also uncommon. As in the story of the development of Keith and Bob's interest in the altitude of pressure, we have seen the question of what the altitude of pressure means, as well as what pressure itself means, come up repeatedly in the social interactions around the use of the Climate Visualizer. Fortunately, there are many experiential referents for climate data such as pressure and altitude (e.g., the effect of pressure through the weight of the water in a swimming pool or ears popping on airplanes or in the mountains). However, these experiences still need to be related back to the data sets if they are to aid in making the data sets and their visualizations intelligible. We address the issue of the meaning of units by providing alternatives that are more likely to be salient to the students' experience when these are available, such as 
temperature in Fahrenheit as well as Celsius, but for pressure, even inches of mercury is not often something students can relate to experience.

Some aspects of the tacit knowledge that atmospheric scientists have are particularly difficult to reify with contextual cues such as those we have employed to provide geographic context. An example is how the data in NMC are collected and processed, which relates to how they may legitimately be used. The NMC data are collected from numerous stations and then integrated with models; hence, a full understanding of where the data come from may be elusive. The NMC data have a granularity of $2^{\circ}$ latitude $\times 4^{\circ}$ longitude, hence they are quite coarse and are somewhat inappropriate to use to report the temperature of a city. Yet reporting the temperature of a city is exactly what many students do in their initial use of these data. This is another example where the Climate Visualizer relies on the social surround, rather than trying to anticipate or limit the students' actions.

The visual representations used in the scientific community and in the Climate Visualizer are sometimes problematic for students as well. For example, high school students are rarely familiar with how to read contour maps. This problem exists on two levels, first, understanding the significance of general patterns within the contour map such as densely packed lines, and second, understanding the significance of specific contour maps for interpreting the data, such as for detecting cyclones using the NMC data sets. In addition, students may have difficulty understanding the SciV's use of colors. ${ }^{9} \mathrm{Re}$ search in color perception and cartography indicates that varying brightness and saturation inconsistently is confusing (Robinson, 1960; Bertin, 1983), yet this is done by many color palettes. Further, our perception of color is variable, hence we perceive the differences between some colors more intensely than others. This can complicate the interpretation of SciVs. Finally, the different variables can use colors in varying ways. For example, if a student picked a rainbow color palette for both the temperature and altitude variables, each color is being mapped to multiple values.

When we have encountered students facing these difficulties with use of the Climate Visualizer, they have been dealt with through learning conver-

\footnotetext{
${ }^{9}$ In addition to the problems enumerated here, color-blind students would obviously face far more serious problems in meaningfully interpreting color representations of data.
}

sations between the students and individuals, such as their teachers or scientists, with more expertise. We hope to continue to refine ways in which the Climate Visualizer can support these conversations, but the need for a social environment that can effectively support such learning conversations is clear. We turn now to a discussion of the design of classroom environments that we believe can optimize opportunities for social support of the Climate Visualizer.

\section{THE CLIMATE VISUALIZER IN THE CLASSROOM ENVIRONMENT}

Achieving authentic scientific practice is a primary concern of the Learning Through Collaborative Visualization Project (CoVis), of which the Climate Visualizer is a part (Pea, 1993a). In this section, we describe the environment provided by the $\mathrm{CoV}$ is classroom and point out how this environment supports the enculturation of students into the practices of science. We first describe the pedagogical approach we have adopted, Project-Enhanced Science Learning. This is followed by a description of the supports for collaborative work provided by the CoVis network testbed and specialized software such as the Collaboratory Notebook, a networked hypermedia data base that provides support for students in the process of scientific inquiry. Finally, we discuss the authenticity of the students' work in this environment incorporating the Climate Visualizer, in light of the learning-theoretic concept of peripheral participation in communities of practice (Lave and Wenger, 1991).

\section{Project-Enhanced Science Learning}

We envision the use of the Climate Visualizer in classrooms using an approach called Project-Enhanced Science Learning (PESL) (Ruopp et al., 1993). In PESL classrooms, students generally work on projects that go deeper and extend over longer periods of time than most traditional classroom activities. The approach is generally most successful when students work on projects that come out of their own questions and interests relating to the field under study. By starting with questions in which the students have a genuine interest, situations that will benefit from using the Climate Visualizer can be identified. This kind of a situation is considered 
preferable to one where there is a set curriculum, since the students may not find such a curriculum motivating, nor is it true to authentic scientific inquiry.

In the process of working on a project that includes refining a research question, obtaining and analyzing data (possibly using the Climate Visualizer), and reporting and defending their findings, many natural opportunities for cognitive apprenticeships through learning conversations and problem solving arise (Collins et al., 1989). These conversations can take place among groups of students as well as groups consisting of students, teachers, and even practicing scientists available through the Internet (see Network Testbed below). The conversations can focus on questions such as the appropriate uses of data and visualizations, the meaning of variables displayed in the visualizations, and the use of evidence to support conclusions. At the workshop Keith and Bob took part in, Stan, Gregg, and the other teachers and workshop facilitators had created an environment where the PESL approach could work.

Projects that involve students' direct participation in acquiring data may also help them clarify the connection between atmospheric phenomena and data, such as that found in the Climate Visualizer. Student data collection can help by relating their experience to data and understanding the problem of experimental accuracy. The experiential nature of data collection can allow for a more direct experience and examination of the data. For example, when students collect temperature readings, they are also experiencing the temperature. In addition, when students collect data, they need to attempt to assess the accuracy and margin of error. When the data fail to meet students' expectations, they must decide whether the data collection is faulty or their hypotheses are in error. The students' personal stakes in the data could enhance their interest in answering this question (Rubin, personal communication; Confrey, 1993). The combination of student-collected data and the data in the Climate Visualizer within the context of projects should prove powerful. In fact, examining both student-collected data and data from outside sources has been recommended to enrich understanding of the relationships between phenomena, data, and models (Newman and Torzs, 1991).

\section{Supports for Collaborative Work}

The work of modern science is seldom an isolated effort. Science has always included a great deal of collaborative work, and today a great deal of this collaboration involves people separated over distance and time (Finholt and Sproull, 1990; D'Amico et al., 1994). Thus, it is appropriate to include a focus on collaborative work as part of our efforts to help students emulate and critique the practices of science.

\section{Network Testbed}

As part of the CoVis project (Pea, 1993a), students will have access to a number of communications media for use in collaborative project work. This includes traditional low-bandwidth technologies such as e-mail and netnews, and also newer highbandwidth options such as video teleconferencing and shared computer screens (see the Appendix for technical details and Fish et al., 1992). These media provide opportunities for learning conversations that extend over time and space, from the two participating Illinois high schools to Northwestern University, to the Department of Atmospheric Sciences at University of Illinois/Urbana-Champaign, the Exploratorium Science museum in San Francisco, and other scientists on the Internet.

The fact that students in CoVis are gaining access to practicing research scientists is particularly important. Practicing atmospheric scientists, who otherwise would not be available in the classroom, can provide modeling and coaching (Collins et al, 1989 ) that goes beyond that available from teachers who are not currently doing scientific research in these fields. While we do not underestimate the social complexities of such encounters, this opportunity for the students to "talk science" (Lemke, 1990) with these scientists should help them gain an understanding of how experts analyze and discuss scientific problems and give them a glimpse into the world of scientific research labs.

\section{Collaboratory Notebook}

The Climate Visualizer is integrated with an inscriptional data base, called the Collaboratory Notebook, to help students carry out project work and appropriate the practices of scientists. The Col- 
laboratory Notebook provides a shared medium for recording and reflecting on scientific inquiry. This includes embedding SciVs from the Climate Visualizer and other tools (for instance real-time weather maps) with accompanying textual commentary. These inscriptions can reference each other in a structured hypermedia environment, thus enabling collaborative work, competitive argumentation, and meaningful negotiation between students engaged in a knowledge-building community of inquiry (Edelson and O'Neill, 1993; O'Neill and Gomez, 1993; for related work, see Scardamalia and Bereiter, 1991).

The Collaboratory Notebook supports the production of inscriptions that reference, modify, critique, affirm, and extend other inscriptions. The goal of this communal data base is to support students' efforts to produce inscriptions that do not stand alone, but rather refer to the inscriptions of their peers and of other scientists. In addition, the communal data base provides support for the identification of specific forms of scientific argumentation and inquiry that relate to one another. Thus, sections can be identified as questions, conjectures that attempt to answer those questions, evidence for and against a conjecture, or plans for some aspect of project work such as the construction of a series of SciVs. References from one entry to another serve as hypertextual links; thus, they can be used to retrieve the referenced section. This same mechanism is used to link SciVs to textual entries. When a SciV is retrieved, it is shown in one of the small static windows described above. However, if the student wants to alter the SciV it can be activated. This places it in the large window, thus making available the full set of crafting tools. Hence, the diagrams of these scientific inscriptions are active, in that the data behind them are present and the visual representation of the data can be altered to highlight other aspects or alternative similar data shown in the same representation. This provides the student commentators with a powerful mechanism for checking and responding to the results and rhetoric that were expressed by their fellow students or scientists.

The Collaboratory Notebook includes specific affordances and mechanisms that support collaborative work. Each entry is identified by the individual or group that created it. Multiple students can join a session to author notes and construct SciVs. Any entry can be designated as private to an individual or group or open to all readers. Thus, individuals can keep a private journal on line, groups can keep a communal scientific notebook detailing an inquiry, and public discussions of general interest issues can be held.

There are a number of open questions concerning the maintenance of the Collaboratory Notebook: Who provides the role of an editor that prunes away the detail to allow the major issues to shine through? How is consensus developed after open debate has been pursued? Like the Climate Visualizer, this tool and questions regarding its use in science education will be refined through our classroom experiences with the CoVis software.

\section{How Authentic is the Learning Environment to Scientific Practice?}

As the above learning environment incorporating the Climate Visualizer is put into place, we may ask which of its aspects are consistent with authentic scientific practice (Brown et al., 1989). Further, what problems can we expect to occur in the process of leading students into the community of science as peripheral members?

The Climate Visualizer aims for authenticity in helping students use SciVs, including the data shown and representation used to visualize them (e.g., raster images). In order to accomplish this, we have deemed it prudent to perform much of the work of assembling a SciV, including making default choices based on our knowledge of scientific practices so as to prevent certain errors, such as nonsensical combinations of data. Errors and inappropriate choices made by students could provide for beneficial learning conversations and insights, but we have prevented some of these in hopes of allowing for greater emphasis on higher-level issues in the domain, rather than floundering amid details. On the other hand, since we recognize that the choices of representation to use in viewing data are an important aspect of learning about SciVs, we have allowed for greater freedom along some dimensions (e.g., the choice of color palette).

In any scientific endeavor, the kinds of inquiry possible are limited by the data and tools available. In the Climate Visualizer, we have assembled a cognitive tool that we believe will support in-depth scientific inquiry in climatic and meteorological topics, but this inquiry will at times be constrained by the data we have on-line and the operations we provide. Further use of the Climate Visualizer in classroom 
settings will help inform our future design decisions along dimensions such as these.

Some of the conditions that affect a scientist's motivations and ability to pursue scientific research are difficult for us to ameliorate directly through design of software or activities. Two examples are selfregulation of time and financial reward. In most schools, students' time is highly regulated by subject and broken into very small sections, usually less than an hour in length. This is known to be problematic for project-oriented science learning (Ruopp et al., 1993). Students receive no financial compensation for their efforts, although they might predict eventual reward. Other motivations differ as well. Scientists often aim to achieve fame as reward for their work. Students rarely achieve notoriety reaching beyond their peers, although for a select few scholarships and prizes promote them into an elite circle. In contrast, scientists have established means of gaining fame, many of them revolving around publishing their work in journals. If the Collaboratory Notebook were widely deployed and questions of scale-up successfully addressed, then students too might form a community that can provide recognition for its members. Finally, scientists are, in general, articulated intellectually, far in advance of most students. This manifests itself in extensive knowledge of areas such as mathematics and the various branches of science and a sharp inclination to immerse themselves in intellectual problems. Students do not have this base of knowledge nor can we assume that they have these problem-solving habits. The Climate Visualizer is designed to indirectly ameliorate such difficulties by enhancing student accessibility - the simplified interface and limited control over choice of representations should help students who do not have the time, motivation, and intellectual background to obtain access to SciV.

\section{FUTURE WORK AND CONCLUSIONS}

In our judgment, scientific visualization can provide science students with a vehicle for approaching scientific phenomena that have been inaccessible to direct experience. Like the microscope, which transforms minute phenomena into "human-sized" pictures, SciVs can present data within the frame of a computer screen that spans the entire world, the galaxy, or even the universe. Moreover, depending on one's purposes, these images can be manipulated, they can be magnified in scale, and their underlying data can be smoothed or combined with other data. The synoptic view provided by such visualizations, combined with the capacities of visualization tools for exploring the meanings of SciVs by manipulating them in structured ways, provides students with a way to experience and understand previously obscure phenomena. As students explore the SciVs and negotiate their meanings with fellow students or more expert teachers and scientists, their view of the SciVs and the physical world they are abstracted from may be transformed. Graphical representations of features of the physical world or models are observed and named through directed investigation and discussion, and the connections between the SciV and the originating phenomena are explored. In this way, previously inaccessible phenomena can be studied through exploratory learning and problem-solving learning conversations that arise within project investigations.

It is a fundamental premise of the Learning through Collaborative Visualization Project, of which the Climate Visualizer is a part, that scaffolded learning environments are needed to help students in this process of appropriating SciVs for the purposes of engagement and learning of science. These scaffoldings may be provided in part by designed properties of the human-computer interface; in our case, considerable knowledge engineering work with scientific experts in the atmospheric sciences led us to acknowledge the tacit knowledge that they utilize in working with existing visualization software tools that we then needed to build into the software as front-end constraints, or scaffolds, to support student choice of meaningful operations. SciVs can become meaningless when their explanatory context is missing or through inappropriate operations. The Climate Visualizer described in this paper assists the student by placing the foreground SciVs into a background of supporting and linked representations, while providing for the specification and execution of structured manipulations and operations.

The design of such a learning environment demands a multidisciplinary team. In particular, the practices of scientists must be observed and analyzed in a process similar to the observations of a sociologist. In addition, the construction of the software artifact draws on expertise in computer science, human-computer interactions and graphics design, and subject matter expertise. Once built, the software needs to be evaluated by students and teachers within classrooms, thus employing the skills of edu- 
cators and social scientists. Increasingly, team approaches of this kind are required to build learning and work environments that incorporate cognitive technologies based on expert practice. We believe such collaborative efforts are necessary to the successful design of learning environments that attempt to enculturate students into scientific communities. The Climate Visualizer has benefited from the mutual interaction of experts in all of the above fields.

Scaffolding support in science learning environments must also be social in nature, since the technology is foremost an interpreted artifact, whose patterns of use and range of appropriateness are determined in communities of practitioner use. The assemblage of a networked community of students, teachers, and scientists connected by the shared goals of the Collaborative Visualization Project provides the opportunity to promote and evaluate the use of SciVs as inscriptions in pursuit of distributed scientific inquiry. The six teachers participating in this phase of the CoVis Project are each attempting to use project-enhanced science learning to encourage more in-depth student inquiry and to determine the nature of the social support for creating project teams, distributed learning groups, and fostering students' conceptual learning in this subject domain. The members of the CoVis community have at their disposal the Climate Visualizer and its surrounding social and technological environment in support of these efforts. A keen awareness of the role of inscriptions and their use in negotiating meaning can benefit this community. For example, by using the Collaboratory Notebook, reviewers (whether peers, teachers, or mentors) can access students' or scientists' arguments along with the SciVs they employed. In critiquing these arguments, the SciVs can be reinspected, their data analyzed differently, and the reviewers' comments and their SciVs entered into the same hypermedia web. Hence, with the integration of collaborative learning software and SciV tools and data, we have sought to provide explicit support for the interactional negotiation of meaning around inscriptions by scientists and students. We look forward to examining how students engaged in this environment for day-to-day classroom activities are able to appropriate SciVs using the affordances that have been designed.

There remain a number of directions for future work. For instance, even though this is already a substantial challenge, given the magnitude of such data, the Climate Visualizer focuses only on SciVs of climatic data sets. We believe the educational community could greatly benefit from the design of learning environments for other domains. Some investigative work along these lines is already underway (Greenberg et al., 1993; Koker and Rowe, 1993; Weather Underground, 1993), including another $\mathrm{CoV}$ is Project-developed weather visualizer (Fishman and D'Amico, 1993). A more ambitious longerterm goal is a domain-independent framework for facilitating access to a wide variety of scientific data sets. However, further work is required to understand the commonalities and differences between domains. Further, we have attempted to scaffold several process functions within the Climate Visualizer, but have not at this stage of our work scaffolded motivation or problem-solving strategies directed specifically at climatology. Future researchers might find ways to build support for such goals more directly into their learning environments. One promising direction for this is to provide "macrocontexts" for the students' activity, similar to the Jasper series of the Cognition and Technology Group at Vanderbilt University (Cognition and Technology Group at Vanderbilt, 1990, 1992).

The explicit goal of our work with the Climate Visualizer and future extensions is to make the community of science and its means of discourse accessible to a much broader group of people. The proper subject of science is the understanding of our physical world. Coupled with this understanding comes an informed ability to choose where and how to effect change to this environment. Recent advances in remote-sensing technology and its deployment are enabling the acquisition of increasingly detailed descriptions of our environment (Zorpette, 1993). If this information can be broadly distributed and understood, it could have a profound impact on the decisions we make. Unfortunately, the knowledge required to understand these data sets is often quite tacit and accessible to only small communities of scientists. The Climate Visualizer represents an attempt to make more of this tacit scientific knowledge explicit and to provide powerful visualization affordances, thus lowering the "entrance fee" to the scientific community and its new lenses for understanding the physical world. Similarly, with the integrated use of the Collaboratory Notebook with the Climate Visualizer, we seek to scaffold the social interchanges involving use and interpretation of SciVs that are essential to entering into the language game of scientific visualization. 
Given the promise we hope to have demonstrated that these new tools hold for science education, achieved by using increasingly accessible high-performance computer and communications technologies, we encourage others to join our efforts in helping students experience nature and its interpretation through the artifice of scientific visualization and jointly construct knowledge through collaborative communication tools.

Acknowledgments - We are grateful for research support of the CoVis Project by the National Science Foundation grant MDR-9253462, by Apple Computer, Inc., External Research, by Sun Microsystems, and by our industrial partners Ameritech and Bellcore. We would also like to thank our colleagues from the CoVis Project and community of users for extended discussions of these issues and continual useful feedback on design, rationale, and pedagogical issues. Special thanks to climatology Professor Raymond T. Pierrehumbert for his initiation and support of our entries into the wide world of climate data sets and tools and to Elliot Soloway for invaluable design advice. In addition, we are indebted to our two anonymous reviewers at the Journal of Science Education and Technology who suggested a better organization for our material.

\section{APPENDIX: TECHNICAL DESCRIPTION OF THE CLIMATE VISUALIZER AND COVIS ENVIRONMENT}

A technical description of the Climate Visualizer is provided in order to more fully characterize it, to discuss design trade-offs, and to indicate the amount of effort that went into its creation.

The Climate Visualizer runs on the Apple Macintosh computer and was written in Apple's implementation of Common Lisp using the CLOS standard. Currently, the data sets for the Climate Visualizer are retrieved from a hard disk shared with a Sun Microsystem UNIX computer. If the requested data set is not present, a remote shell command is executed via the TCP/IP network protocol to the Sun in order to retrieve the data set from the CD-ROM. The appropriate scientific visualizations are then performed by a commercial application called Spyglass Transform; this application is controlled through the use of Apple Events. The visualizations are then imported to the Climate
Visualizer, which displays them to the user. In addition, the data set itself is imported so the values can be easily queried. While the visualizations are stored in files, the textual entries are stored and retrieved from an Oracle relational data base, thus providing multiple-reader, single-writer access.

The two technologies enabling collaboration with experts through high-speed two-way video links and shared computer displays are called Cruiser and Timbuktu. Cruiser is a video teleconferencing system, developed by Bellcore that runs over publicly available ISDN phone lines. Timbuktu is a commercial program from Farrallon that enables two computers to share one screen, with full editing capabilities, across a distance, again over commercial phone lines. We have Cruiser stations next to CoVis computers running Timbuktu in our classrooms at the two high schools, in labs at Northwestern, and in the offices of our project partners at the University of Illinois/Urbana-Champaign's Department of Atmospheric Sciences and the Exploratorium Science Museum in San Francisco, California.

As the above architecture illustrates, we have attempted to build our efforts on top of standard tools and platforms whenever possible, thus providing for quick development and later scale-up. However, with these prepackaged tools also come limitations. For example, Spyglass Transform does not support three-dimensional data sets. Another current limitation is that we presently only support the NMC data sets.

\section{REFERENCES}

Bertin, J. (1983). A new look at cartography. In Taylor, D. R. F. (Ed.), Progress in Contemporary Cartography, Volume II: Graphic Communication and Design in Contemporary Cartography, John Wiley and Sons, Chichester.

Borroughs, W. J. (1991). Watching the World's Weather, Cambridge University Press, Cambridge.

Brodie, K. W., Carpenter, L. A., Earnshaw, R. A., Gallop, J. R. Hubbold, R. J., Mumford, A. M., Osland, C. D., and Quarendon, P. (1992). Scientific Visualization, Springer-Verlag, Berlin.

Brown, J. S., Collins, A., and Duguid, P. (1989). Situated cognition and the culture of learning. Educational Researcher 18(1): 32-42.

Cognition and Technology Group at Vanderbilt. (1990). Anchored instruction and its relationship to situated cognition. Educational Researcher 19(6): 2-10.

Cognition and Technology Group at Vanderbilt. (1992). The Jasper Series as an Example of Anchored Instruction: Theory, Program Description, and Assessment Data. Educational Psychologist 27(3): 291-315.

Collins, A., Brown, J. S., and Newman, S. E. (1989). Cognitive apprenticeship: teaching the crafts of reading, writing, and 
mathematics. In Resnick, L. B. (Ed.), Knowing, Learning, and Instruction: Essays in Honor of Robert Glaser, Lawrence Erlbaum Associates, Hillsdale, New Jersey, pp. 453-494.

Confrey, J. (1990). A review of the research on student conceptions in mathematics, science and programming. In Cazden, C. (Ed.), Review of Research in Education, Vol. 16, American Educational Research Association, Washington, DC, pp. 3-56.

Confrey, J. (1993). Splitting vs. Counting: New Approaches to Multiplication and Exponential Functions. Paper presented at the Institute for the Learning Sciences, Northwestern University, February 26, 1993.

D’Amico, L., Fishman, B., Gordin, D. N., McGee, S., O'Neill, K., and Polman, J. (1994). The atmospheric sciences: Six views into a community of practice (in preparation).

Dewey, J. (1956). School and Society, University of Chicago Press, Chicago (originally published 1899).

Edelson, D. C., and O'Neill, D. K. (1994). The CoVis Collaboratory Notebook: Supporting collaborative scientific enquiry. Conference Proceedings-National Educational Computing Conference, Boston, MA, June 13-15 1994, pp. 146-152.

Finholt, T., and Sproull, L. S. (1990). Electronic groups at work. Organizational Science 1: 41-64.

Fish, R. S., Kraut, R. E., Root R. W., and Rice, R. E. (1992). Evaluating Video as a Technology for Informal Communication. Bellcore Technical Memorandum No. TM-TSV019988.

Fishman, B. J., and D'Amico, L. (1994). Which Way Will the Wind Blow? Networked Computer Tools for Studying the Weather. Ed-Media 94 (submitted).

Gibson, J. J. (1977). The theory of affordances. In R. E. Shaw and J. Bransford (Eds.), Perceiving, Acting, and Knowing, Erlbaum Associates, Hillsdale, N.J.

Gordin, D. N., and Pea, R. D. (1994). Scientific visualization as an educational technology. The Journal of the Learning Sciences 4(3).

Greenberg, R., Kolvoord, R. A., Magisos, M., Strom, R. G., and Croft, S. (1993). Image processing for teaching. Journal of Science Education and Technology 2(3): 469-480.

Kaufman, W. J., III, and Smarr, L. L. (1993). Supercomputing and Transformation of Science, Scientific American Library, New York.

Koker, T., and Rowe, B. (1993). ChemViz: An NSF sponsored program to bring High Performance Computing to America's high schools. Unpublished manuscript.

Latour, B. (1988). Drawing things together. In Lynch, M., and Woolgar, S. (Eds.), Representation in Scientific Practice, MIT Press, Cambridge, Massachusetts.

Latour, B., and Woolgar, S. (1979). Laboratory Life: The Construction of Scientific Facts, Princeton University Press, Princeton, New Jersey.

Lave, J. (1990). The culture of acquisition and the practice of understanding. In Stigler, J. W., Shweder, R. A., and Herdt, G. (Eds.), Cultural Psychology: Essays on Comparative Human Development, Cambridge University Press, Cambridge, pp. 309-327.

Lave, J., and Wenger, E. (1991). Situated Learning: Legitimate Peripheral Participation, Cambridge University Press, Cambridge.

Lemke, J. L. (1990). Talking Science: Language, Learning, and Values. Ablex, Norwood, New Jersey.

Linn, M. C., diSessa, A., Pea, R. D., and Songer, N. B. (1994). Can research on science learning and instruction inform standards for science education? Journal of Science Education and Technology (in press).

Lynch, M., and Woolgar, S. (1988). Introduction: Sociological orientations to representational practice in science. In Lynch, M., and Woolgar, S. (Eds.), Representation in Scientific Practice, MIT Press, Cambridge, Massachusetts.
McCormick B. H., DeFanti, T. A., and Brown, M. D. (Eds.). (1987). Visualization in scientific computing. Computer Graphics 21: 6.

McGee, S., and Pea, R. P. (1994). Cyclone in the classroom: Bringing the atmospheric science community into the high school. Proceedings of the 74th Annual Meeting of the American Meteorological Society, January 23-28, 1994, Nashville, Tennessee (in press).

Newman, D., and Torzs, F. (1991). A world in the classroom: Making sense of seasonal change through talk and technology. Technical Report No. 11, Center for Technology in Education, Bank Street College of Education.

Newman, D., Crowder, E., and Morrison, D. (1994). Scientific sense-making in elementary classroom conversations (in preparation).

Office of Science and Technology Policy (1991). Grand Challenges: High Performance Computing and Communications: The FY 1992 U.S. Research and Development Program. A Report by the Committee on Physical, Mathematical, and Engineering Sciences, Federal Coordinating Council for Science, Engineering, and Technology, Executive Office of the President, Washington, D.C.

Office of Science and Technology Policy (1993). High Performance Computing and Communications: Toward a National Information Infrastructure: The FY 1994 U.S. Research and Development Program. A Report by the Committee on Physical, Mathematical, and Engineering Sciences, Federal Coordinating Council for Science, Engineering, and Technology, Executive Office of the President, Washington, D.C.

O'Neill, D. K., and Gomez, L. (1994) The Collaboratory Notebook: A Networked Knowledge-Building Environment for Project Learning. In Ottman, T. and Tomek, I., Proceedings of ED-MEDIA 94-Word Conference on Educational Multimedia and Hypermedia, pp. 416-423.

Pea, R. D. (1985). Beyond amplification: Using computers to reorganize human mental functioning. Educational Psychologist 20: $167-182$.

Pea, R. D. (1987). Cognitive technologies for mathematics education. In Schoenfeld, A. (Ed.), Cognitive Science and Mathematics Education, Lawrence Erlbaum Press, Hillsdale, New Jersey, pp. 89-122.

Pea, R. D. (1992). Augmenting the discourse of learning with computer-based learning environments. In de Corte, E., Linn, M., Mandl, H., and Verschaffel, L. (Eds.), ComputerBased Learning Environments and Problem-Solving, Springer Verlag, New York, pp. 313-343.

Pea, R. D. (1993a). The collaborative visualization project. Communications of the ACM 36(5): 60-63.

Pea, R. D. (1993b). Practices of distributed intelligence and designs for education. In Salomon, G. (Ed.), Distributed Cognition. Cambridge University Press, New York.

Pea, R. D., and Kurland, D. M. (1987). Cognitive technologies for writing development. In Frase, L. (Ed.), Review of Research in Education, Vol. 14, AERA Press, Washington, D.C., pp. $71-120$.

Rheingold, H. (1985). Tools for Thought: The History and Future of Mind-Expanding Technology, Simon and Schuster, New York.

Robinson, A. H. (1960). Elements of Cartography, John Wiley and Sons, New York.

Roschelle, J. (1990). Designing for conversations. AAAI Symposium on Knowledge-Based Environments for Learning and Teaching, Stanford, CA.

Ruopp, R., Gal, S., Drayton, B., and Pfister, M. (1993). LabNet: Toward a Community of Practice. Lawrence Erlbaum Press, Hillsdale, New Jersey.

Scardamalia, M., and Bereiter, C. (1991). Higher levels of agency for children in knowledge building: A challenge for the de- 
sign of new knowledge media. The Journal of the Learning Sciences 1(1): 37-68.

Schoenfeld, A. (1987). What's all the fuss about metacognition. In Schoenfeld, A. (Ed.), Cognitive Science and Mathematics Education, Lawrence Erlbaum Press, Hillsdale, New Jersey, pp. 189-215.

Short, N. M., Lowman, P. D., Jr., Freden, S. C., and Finch, W. A., Jr., (1976). Mission to Earth: LANDSAT Views the World. National Aeronautics and Space Administration, Washington, D.C.

von Glasersfeld, E. (1987). Preliminaries to Any Theory of Representation. In Janvier, C. (Ed.), Problems of Representation in the Teaching and Learning of Mathematics, Lawrence Erlbaum Press, Hillsdale, New Jersey, pp. 215-225.
Vygotsky, L. S. (1978). Mind in Society: The Development of the Higher Psychological Processes. Harvard University Press, Cambridge, Massachusetts (originally published 1930).

Weather Underground. (1993). Weather as the Paradigm for Instructional Technology. Unpublished manuscript of the University of Michigan, Department of Atmospheric, Oceanic and Space Science.

Winn, W., and Holliday, W. (1982). Design principles for diagrams and charts. In Jonassen, D. H. (Ed.), The Technology of Text: Principles for Structuring, Designing and Displaying Text. Educational Technology Publications, Englewood Cliffs, New Jersey.

Wittgenstein, L. (1967). Philosophical Investigations. Oxford Blackwell (originally published in 1949).

Zorpette, G. (1993). Sensing climate change. IEEE Spectrum 30(7): $20-27$. 